\title{
Effect of etanercept in polymyalgia rheumatica: a randomized controlled trial
}

\author{
Frederik Kreiner*, Henrik Galbo
}

\begin{abstract}
Introduction: To elucidate in polymyalgia rheumatica (PMR) the role of tumor necrosis factor (TNF) $\alpha$ and the therapeutic potential of blockade with soluble TNF- $\alpha$ receptor, we carried out the first randomized controlled trial with etanercept in PMR.
\end{abstract}

Methods: Twenty newly diagnosed, glucocorticoid (GC) naïve patients with PMR and 20 matched non-PMR control subjects completed the trial. Subjects were randomized in a 1:1 ratio to monotherapy with etanercept (25 mg s.c. biweekly) or placebo (saline) for 14 days. Study outcomes were assessed at baseline and after 14 days. The primary outcome was the change in PMR activity score (PMR-AS). Secondary outcomes were: changes in erythrocyte sedimentation rate (ESR) and plasma levels of TNF- $\alpha$ and interleukin (IL) 6; patients' functional status (health assessment questionnaire) and cumulative tramadol intake during the trial.

Results: At baseline, plasma TNF- $\alpha$ was higher in patients than in controls $(P<0.05)$. The concentration always increased with etanercept treatment $(P<0.05)$. In patients, etanercept decreased PMR-AS by $24 \%(P=0.011)$, reflecting significant improvements in shoulder mobility, physician's global assessment and C-reactive protein, and insignificant $(P>0.05)$ improvements in duration of morning stiffness and patient's assessment of pain. In parallel, ESR and IL-6 were reduced $(P<0.05)$. Placebo treatment did not change PMR-AS, ESR and IL-6 $(P>0.05)$.

Functional status did not change and tramadol intake did not differ between patient groups. In controls, no changes occurred in both groups.

Conclusions: Etanercept monotherapy ameliorates disease activity in GC naïve patients with PMR. However, the effect is modest, indicating a minor role of TNF- $\alpha$ in PMR.

Trial registration: ClinicalTrials.gov (NCT00524381).

\section{Introduction}

Polymyalgia rheumatica (PMR) is the most common chronic inflammatory disease in the elderly [1]. Clinically, it is characterized by pain in the neck and lower back as well as proximal extremity complaints, including tender, aching, and stiff muscles. Patients feel fatigued and their level of physical activity is reduced [1]. These symptoms are accompanied by elevated erythrocyte sedimentation rate (ESR) and increased blood levels of C-reactive protein (CRP) [1].

The knowledge of the etiology and pathophysiology of PMR is modest. The prevailing view is that PMR reflects inflammatory processes in synovial membranes in joints,

\footnotetext{
* Correspondence: frederik.kreiner@me.com Department of Rheumatology, Institute of Inflammation Research, Rigshospitalet, Copenhagen University Hospital, Blegdamsvej 9, DK-2100 Copenhagen, Denmark
}

bursae, and tendons [1-4]. Several studies have found elevated blood levels of various proinflammatory cytokines in PMR [5]. Recently, we showed that levels of proinflammatory cytokines, including IL- 6 and TNF- $\alpha$, which both potently induce the expression of acute phase reactants [6] and promote nociception [7,8], are increased in the interstitium of affected muscles [9].

The only effective treatment is medium-dose glucocorticoids (GC), which abolish symptoms within a few days [10]. However, because long-term treatment is necessary, serious adverse effects, including type 2 diabetes, hypertension, and osteoporosis, are frequent [11].

In patients with rheumatoid arthritis (RA), another chronic inflammatory disease and an important differential diagnosis in PMR $[10,12,13]$, administration of TNF- $\alpha$ inhibitors has been a therapeutic success [14]. In PMR, however, no effect of the TNF- $\alpha$ antagonist infliximab 
on relapse frequency and use of prednisone was found in a recent randomized controlled trial (RCT) of newly diagnosed patients [15]. Still, it should be noted that seven clinics participated in that study [15]. It can be expected that a high number of clinics and doctors involved increases the variation associated with clinical evaluation and decisions concerning patients and, accordingly, decreases the ability to detect differences between treatment with TNF- $\alpha$ blockade and placebo. Furthermore, in the mentioned RCT, patients had successfully been treated with prednisone for some weeks before the start of infliximab therapy, which was applied in parallel with a fixed tapering of prednisone treatment [15]. If at all stages the scheduled prednisone dose per se would be sufficient to control the disease, this may have hampered the ability of the study to detect any potential beneficial effect of the added TNF- $\alpha$ blockade.

Finally, even if infliximab has no effect in patients with PMR, the TNF- $\alpha$ antagonist etanercept might still be effective, because the two TNF- $\alpha$ inhibitors act by different mechanisms, being an anti-TNF- $\alpha$ monoclonal antibody and a soluble recombinant Fc-coupled TNF- $\alpha$ receptor fusion protein, respectively. Correspondingly, infliximab and etanercept have different therapeutic potentials in other diseases, for example, only infliximab is effective in granulomatosis disorders such as Crohn's disease and Wegener's granulomatosis [16]. Also, small uncontrolled studies have pointed to a beneficial effect of etanercept in patients with PMR $[17,18]$. Moreover, in a RCT of patients with giant cell arteritis (GCA), which is intimately related to PMR, etanercept was shown to be an effective therapy [19].

As there is a need for effective drugs other than GCs for PMR, and because existing evidence does not exclude a role of etanercept, in the present study we performed the first RCT of etanercept in patients with PMR. The study was a parallel group in a placebocontrolled, double-blinded, RCT with etanercept in a group of newly diagnosed, GC naïve PMR patients and non-PMR control subjects. The trial was carried out at a single center, and all patients were diagnosed and evaluated by the same chief rheumatologist. Furthermore, etanercept was the only anti-inflammatory drug administered. So, the study allows evaluation of the pure effect of etanercept treatment in patients with PMR without any possible blurring interference due to, for example GC treatment. The duration of the trial was 14 days, because for etanercept to be an attractive alternative to GCs in the treatment of patients with PMR, beneficial effects must occur rapidly. Rapid action of an effective anti-TNF- $\alpha$ therapy can also be expected, if TNF- $\alpha$ does in fact play a key role in the pathophysiology of PMR. In agreement with this view, in RA a clear effect of TNF- $\alpha$ antagonism can be expected within a week [20].

\section{Materials and methods Participants}

Patients with suspected PMR were recruited by referral from general practitioners in the Copenhagen municipal area from July 2007 to May 2009. Non-PMR control subjects were recruited by newspaper advertising during the same period. Patients were eligible if they had been diagnosed with PMR according to the Chuang criteria [21], and if they did not fulfill the exclusion criteria, which were: prior or current use of GCs or other immunosuppressive drugs; signs of GCA including cranial symptoms of vasculitis (headache, visual disturbances, jaw claudication, abnormal pulsation or wall of temporal artery, scalp tenderness); infections with systemic impact; hepatitis $\mathrm{B}$ or $\mathrm{C}$ infection; positive tuberculosis screening tests (thorax $\mathrm{x}$-ray imaging, Mantoux skin test and Quantiferon tuberculosis blood test); positive blood or urine culture; uncontrolled diabetes mellitus; uncontrolled hypertension; severe heart failure (New York Heart Association class 3 and 4); other inflammatory diseases than PMR; cancer in the past five years; neuromuscular disease; thyroid disease; disturbance of calcium homeostatis. Control subjects fulfilled the same exclusion criteria as patients and were matched according to sex, age, and body mass index (BMI). Withdrawal criteria were non-compliance, serious exacerbation of the disease, development of serious infections, development of heart failure, and other suspected unexpected serious adverse reactions.

Concurrent use of nonsteroidal anti-inflammatory drugs and GCs was not allowed in both patients and control subjects. One hundred $50 \mathrm{mg}$ tablets of the centrally active opioid-like analgesic tramadol (Mandolgin, Sandoz A/S, Odense, Denmark) were given to the patients, who were instructed to administer them in order to adequately control pain symptoms during the trial (no more than eight tablets per day); patients registered their use of tramadol using a standardized form during the entire trial.

\section{Trial design}

The study was conducted at the Department of Rheumatology at Bispebjerg Hospital, Copenhagen, Denmark as a 14-day single-center, double-blinded, prospective RCT, comparing the effect of 14 days of etanercept treatment $(\mathrm{n}=10)$ against 14 days of placebo treatment $(\mathrm{n}=10)$ in a group of 20 patients with PMR and in an equal sized group of matched control subjects.

The trial was approved by the Danish Medicines Agency (approval number 2612-3497), the Ethical 
Committee (Institutional Review Board, approval number H-D-2007-0040), and by the Danish Data Protection Agency. Furthermore, the trial was entered in the European Eudract database (Eudract number 2007003009-28), and it was registered in the public database clinicaltrials.gov (trial identifier NCT00524381). The study was conducted according to the International Conference on Harmonisation of Good Clinical Practices and was monitored by a good clinical practice monitoring unit before, during, and after the trial period. At the time of screening for trial inclusion, potential participants received thorough written and oral information of the purpose and duration of the trial as well as of predictable adverse events. Before inclusion in the study, all participants signed a written informed consent.

\section{Randomization and blinding}

Following enrollment by a rheumatology specialist (the senior author), participants were randomly assigned to treatment with etanercept or placebo in a 1:1 ratio. A five-block randomization scheme was generated using the web site Randomization.com[22] by two trialassociated senior nurses, who were also responsible for drug preparation and who had no contact with the participants. When included in the trial, subjects were consecutively assigned an identification number according to the randomization scheme by the same two nurses. Subjects who withdrew from the trial were consecutively replaced by new subjects and allocated to the same treatment. Physicians and technicians in direct contact with participants or those responsible for data and plasma analysis including staff that administered the medication were blinded to group assignment. The blinding code was not broken until all trial outcomes had been collected.

\section{Examinations}

One to three days after inclusion in the study, subjects were examined the first of two times, 14 days apart. They were brought by taxi to the laboratory after an overnight fast including abstinence from alcohol and tobacco. Subjects were allowed to take their usual medication, if any, in the morning before the examination but abstained from analgesics. A cannula was inserted into a forearm vein, and subjects rested in a chair 15 minutes before blood samples were drawn. A clinical examination focusing on joint mobility and muscle tenderness was carried out, and a health assessment questionnaire (HAQ; evaluated for use in RA [23]) and some supplementary questions about morning stiffness and daily physical activity were answered. At the end of the first examination, subjects had their first of four injections with trial medication. The last injection was given three days before the final examination (end of study).

\section{Interventions}

Etanercept (Enbrel, Wyeth Pharmaceuticals New Lane, Hampshire, UK) was injected subcutaneously biweekly in the thigh at the standard dose of $25 \mathrm{mg}(1 \mathrm{ml})$. Placebo was $1 \mathrm{ml}$ isotonic saline. Individual injections were given at least $3 \mathrm{~cm}$ apart. To ensure proper blinding, etanercept and placebo, which were both colorless solutions, were prepared in indistinguishable syringes by nurses, who had no interaction with the subjects.

\section{Study outcomes}

The primary outcome was the change in PMR activity score (PMR-AS [24-26]), from baseline to end of study (day 15) in patients and control subjects treated with etanercept or placebo. PMR-AS was calculated from measurements of plasma CRP levels $(\mathrm{mg} / \mathrm{dl})$, the duration of morning stiffness (MST, minutes), the ability to raise the arms (E, 3 to $0: 3$ = no elevation possible; 2 = elevation possible below shoulder height; 1 = elevation possible above shoulder height; 0 = full elevation possible), physician's global assessment (physician's visual analog scale $\left(\mathrm{VAS}_{\mathrm{ph}}\right) ; 0$ to $\left.10 \mathrm{~cm}\right)$, and the patients' assessment of pain (patient's visual analog scale $\left(\mathrm{VAS}_{\mathrm{p}}\right) ; 0$ to $10 \mathrm{~cm}$ ), as [24]:

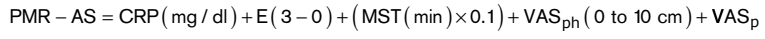

Secondary outcomes were: changes in ESR, cumulative intake of tramadol during the study, and plasma TNF- $\alpha$ and IL- 6 concentrations in all groups at baseline and at the end of the study (day 15). After treatment in patients receiving etanercept, TNF- $\alpha$ concentrations were also measured in immunoglobulin (Ig) G depleted plasma. In patients, functional status was assessed before and after etanercept treatment using the HAQ [23].

To ensure high accuracy of outcome assessments and measurements, all participating staff carefully reviewed the study protocol prior to the start of the trial. Moreover, standard operation protocols were used during all trial-related activities.

\section{Safety and adverse events}

Assessment of the subjects' safety included registration of all adverse events as well as careful examination and questioning of subjects before each injection and/or examination.

\section{Plasma samples}

Blood samples were drawn in stock Vacutainers (Becton Dickinson, Brpndby, Denmark) with EDTA anticoagulant and the proteolysis inhibitor Trasylol (Bayer AG, 
Leverkusen, Germany). Plasma was harvested by centrifugation at $1,200 \mathrm{rpm}$ at $4^{\circ} \mathrm{C}$ for 15 minutes and immediately frozen at $-80^{\circ} \mathrm{C}$ until analysis.

\section{Analytical methods}

ESR and blood CRP were measured at an ISO-certified (ISO 15189:2008) clinical laboratory using the Westergen method (Becton Dickinson, BD Sedi-15) and colorimetric slide tests (measurement range: 5 to $90 \mathrm{mg} / \mathrm{l}$; intra- and interassay coefficients of variation $(\mathrm{CV}): 2.2 \%$ and $10.0 \%$, respectively), respectively. Plasma TNF- $\alpha$ and IL- 6 were analyzed using the Luminex 100 platform (Ramcon, Birkeroed, Denmark); specific kits used were Milliplex (Millipore, ElectraBox Aps, Roedovre, Denmark). Detection levels and intra- and interassay $\mathrm{CVs}$ were: $\mathrm{TNF}-\alpha 0.05 \mathrm{pg} / \mathrm{ml}, 3.5 \%$ and $3.8 \%$, respectively; IL-6 $0.79 \mathrm{pg} / \mathrm{ml}, 13.6 \%$ and $13.3 \%$, respectively. Analyses were carried out on unprocessed plasma. Furthermore, TNF- $\alpha$ was also determined in plasma processed by protein G-based spin columns (Albumin and IgG Depletion Spin Trap, GE Healthcare, Uppsala, Sweden), which remove IgGs including free etanercept and etanerceptTNF- $\alpha$ complexes. To compensate for loss in the columns of free TNF- $\alpha$ and of proteins influencing standard curves, standards dissolved in plasma analogue (matrix included in the MilliPlex kit) were also passed through the spin columns.

\section{Statistics}

We estimated the sample size based on an effect size on PMR-AS of $\rho=|0.75|$. Thus, at an $\alpha$-level of 0.05 and at a statistical power of $80 \%$ (in two-tailed testing), the minimum sample size should be nine subjects in each treatment arm.

Statistical analysis was performed using SPSS 17.0.1 for Mac (SPSS Inc., Chicago, IL, USA). Unless otherwise stated, data are mean \pm standard error of the mean. A two-way analysis of variance was used to determine if data differed between patients and control subjects, if changes occurred with treatment, and if there was any interaction between group and treatment. $P$-values below 0.05 in two-tailed testing were considered significant.

\section{Results}

Twenty-two patients with PMR were included in the trial; of these, two patients, both receiving placebo, withdrew from the study (Figure 1). Twenty-one control subjects without PMR were included; one control subject receiving etanercept withdrew (Figure 1). Baseline anthropometric and clinical findings did not differ $(P>$ 0.05 ) between patients who received etanercept and patients who received placebo (Figures 2 and 3, and Table 1). Nor did these variables differ between control subjects receiving etanercept and placebo, respectively (Table 2, $P>0.05$ ). Age, BMI, and blood pressure did not differ $(P>0.05)$ between patients and control subjects; before treatment all other measurements differed significantly $(P<0.001$ to 0.01$)$ between these groups (Tables 1 and 2, and Figure 3).

After the 14 days of trial participation, all patients were treated with $20 \mathrm{mg} /$ day prednisolone. Within a week, prednisolone abolished any remaining PMR symptoms and normalized CRP and ESR in all patients, supporting the PMR diagnosis.

\section{Primary outcome: PMR-AS}

At baseline, PMR-AS did not differ between patients treated with etanercept and patients treated with placebo (Figure 2, $P>0.05$ ); it was, however, significantly higher in patients than in control subjects (Figure 2, $P<$ 0.0001 to 0.01 ). In all control subjects, PMR-AS did not differ significantly from zero (Figure $2, P>0.05$ ).

During the 14 days of etanercept treatment, PMR-AS significantly decreased by $24 \%$ ( $95 \%$ confidence interval: 12 to $33 \%$ ) in patients (Figure 2), but remained significantly higher in patients compared with control subjects (Figure 2, $P<0.0001$ to 0.01 ). In patients receiving placebo, PMR-AS did not change $(P>0.05)$.

\section{Secondary outcomes}

In patients with PMR, CRP, E and $\mathrm{VAS}_{\mathrm{ph}}$ decreased significantly during etanercept treatment (Figure 3), whereas during placebo treatment only CRP decreased and to a lesser extent $(P<0.05$; Figure 3$)$. Also the other components of the PMR-AS (MST and VAS ${ }_{\mathrm{p}}$ ) tended to decrease during etanercept treatment; however, the changes were not statistically significant (Table 1 , $P>0.05$ ). ESR (Figure 3) as well as IL-6 (Figure 4), which at baseline were markedly higher in patients than in control subjects, decreased in PMR patients receiving etanercept, but not $(P>0.05)$ in patients receiving placebo. The cumulative intake of tramadol (Table 1) was $17 \%$ lower in patients treated with etanercept compared with placebo, but the difference was not significant $(P>0.05)$. HAQ variables did not change in patients receiving etanercept or placebo (data not shown). By the end of the trial, the various variables still did not differ significantly between patients receiving etanercept and placebo $(P>0.05)$. However, the mean IL-6 concentration was markedly higher in patients receiving placebo, and this difference was of borderline significance (Figure 4). Furthermore, all disease parameters were still significantly higher compared with values in control subjects $(P<0.05)$. In control subjects, no changes were seen after either etanercept or placebo treatment.

At baseline, plasma TNF- $\alpha$ concentrations were significantly higher in patients than in control subjects (Figure 5). 


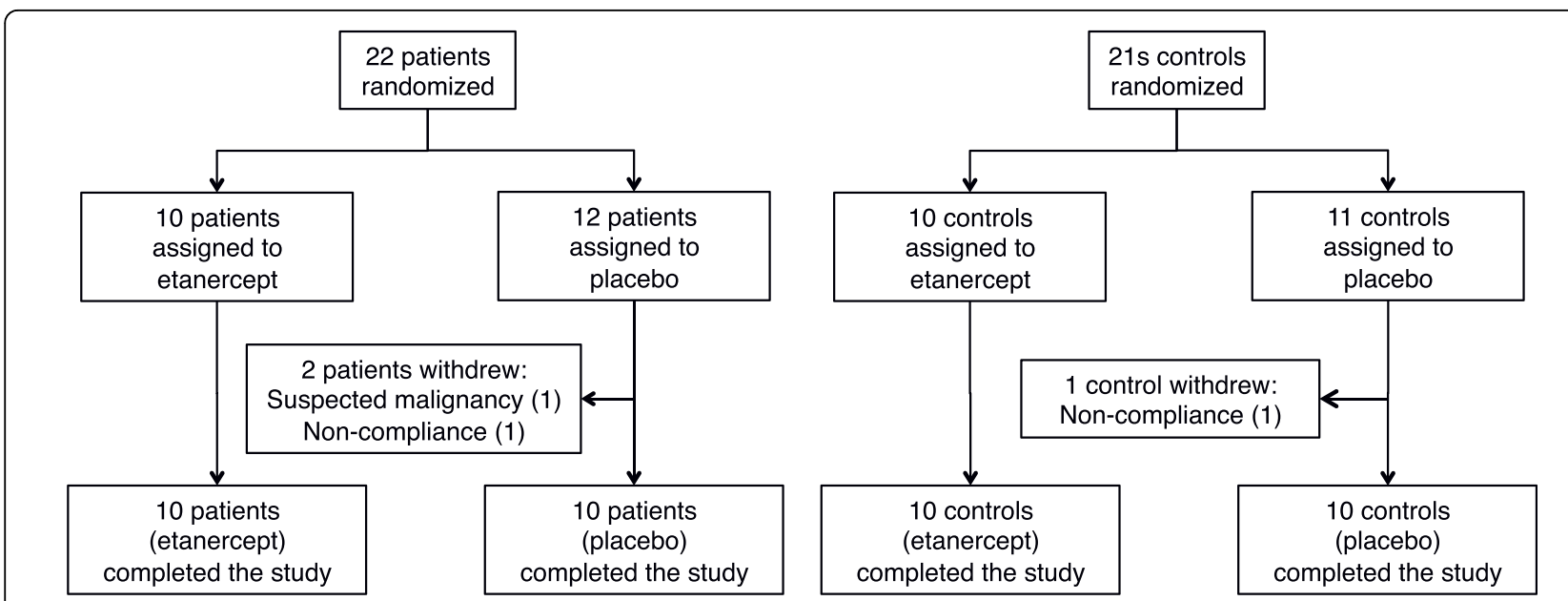

Figure 1 Flow of patients and controls through the trial. This includes randomization, withdrawal numbers and reasons, and number of participants, who completed the study.

During etanercept treatment TNF- $\alpha$ concentrations markedly increased in both groups (Figure 5). During placebo treatment, TNF- $\alpha$ concentrations in plasma decreased in patients but stayed constant in control subjects (Figure 5). The high concentrations seen in patients treated with etanercept were reduced by $61 \%(P=0.02)$ by IgG depletion of plasma, and became similar to concentrations in placebo-treated patients (values before vs after IgG depletion in etanercept $(\mathrm{n}=10)$ and placebo $(\mathrm{n}=10)$ treated

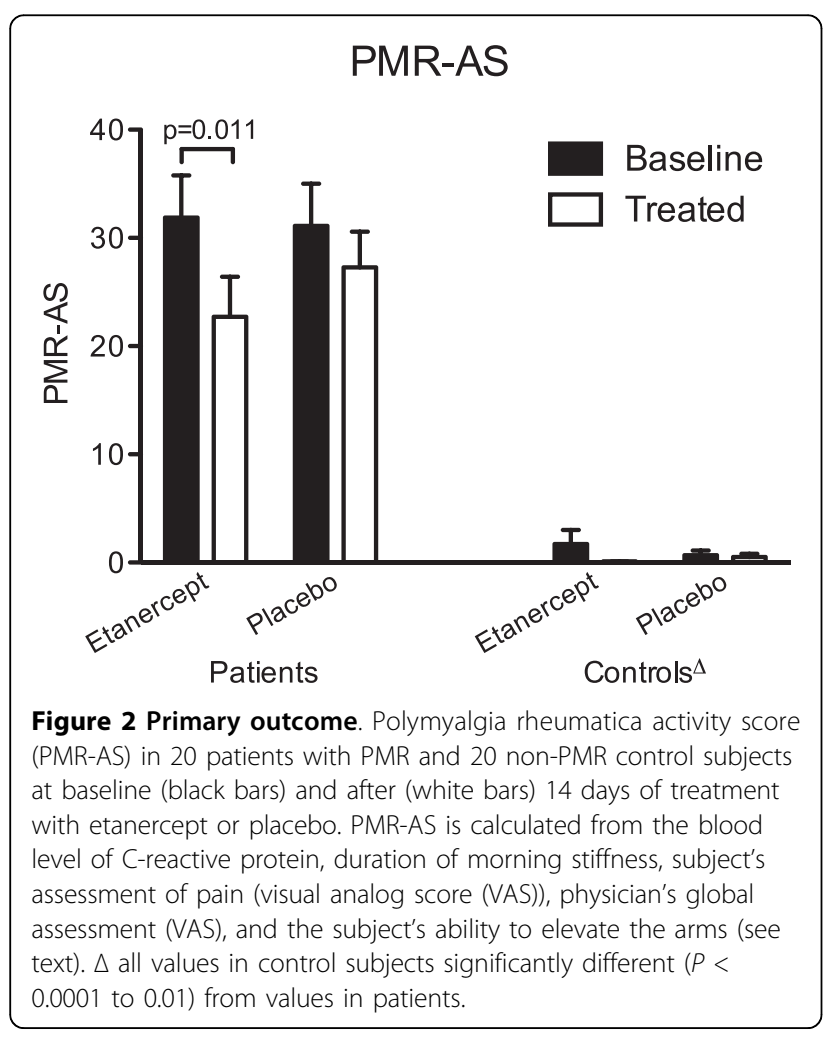

patients, respectively: $16.9 \pm 2.6 \mathrm{pg} / \mathrm{ml}$ vs $10.4 \pm 1.3 \mathrm{pg} / \mathrm{ml}$; $7.1 \pm 0.7 \mathrm{pg} / \mathrm{ml}$ vs $11.5 \pm 1.3 \mathrm{pg} / \mathrm{ml})$.

\section{Safety and adverse events}

Generally, etanercept treatment was well tolerated in all subjects. Two patients and one control subject, all treated with etanercept, had minor local injection-site reactions (rashes). One control subject treated with placebo reported an unsuspected feeling of fatigue. No suspected unexpected serious adverse reactions were observed in any of the subjects.

\section{Discussion}

The present study is the first RCT of the effect of etanercept in patients with PMR. The major finding is that 14 days of etanercept monotherapy ameliorates disease activity in newly diagnosed, GC-naïve patients with PMR. However, the effect is modest indicating that TNF- $\alpha$ does not have a predominant role in the pathophysiology of PMR.

The positive effect of etanercept is evident from the 24\% reduction of PMR-AS in patients treated with the drug, as well as the insignificant changes in PMR-AS observed in placebo-treated patients (Figure 2). The reduction of PMR-AS in patients treated with etanercept reflected decreases in all paraclinical as well as objective and subjective clinical components of PMR-AS. Among these, statistically significant improvements in shoulder mobility (E), physician's global assessment (VAS $\mathrm{Vh}_{\mathrm{ph}}$ ) and CRP were seen, and these changes were paralleled by a significant decrease in ESR and plasma IL-6 concentration (Figures 3 and 4). The fact that both etanercept and placebo had no effect in non-PMR control subjects (Figures 2 to 4 , and Table 2) shows that the significant effects of etanercept seen in patients with PMR were indeed disease specific. 


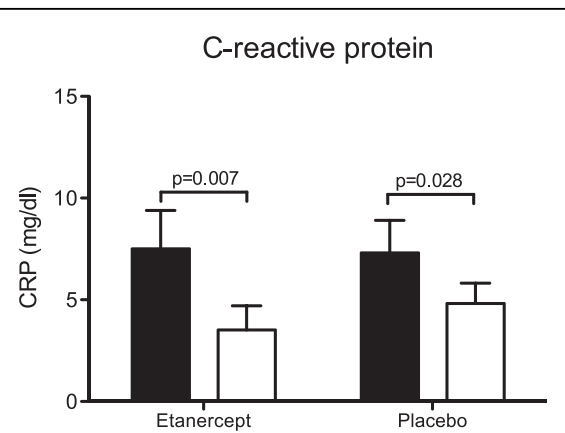

Elevation of arms

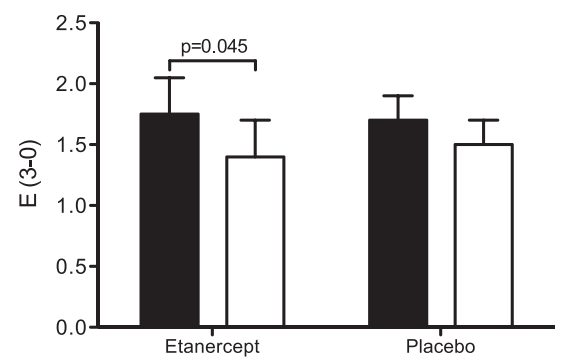

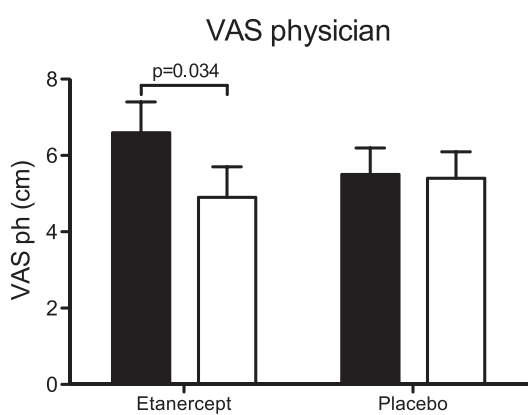

Erythrocyte sedimentation rate

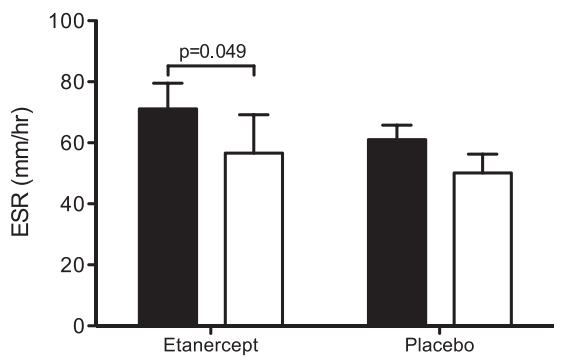

Figure 3 Clinical and paraclinical measurements in patients with polymyalgia rheumatica at baseline (black bars) and after (white bars) 14 days of treatment with etanercept or placebo. C-reactive protein (CRP), patients' ability to elevate the arms (E, 3 to $0: 3=$ no elevation possible; 2 = elevation possible below shoulder height; 1 = elevation possible above shoulder height; 0 = full elevation possible), the physician's global assessment (visual analog score, VAS), and erythrocyte sedimentation rate (ESR).

However, the view that the effect of etanercept is modest in patients with PMR is suggested by the fact that the reductions in both MST and $\mathrm{VAS}_{\mathrm{p}}$ were not statistically significant (Table 1). Furthermore, at the end of the 14 days of etanercept treatment, PMR-AS was still not only higher than in control subjects but also not significantly different from values in placebotreated patients (Figure 2). Correspondingly, although the tramadol intake was lower in etanercept compared with placebo-treated patients, the difference was not

Table 1 Characteristics of patients at baseline and after etanercept/placebo treatment

\begin{tabular}{|c|c|c|c|c|}
\hline & $\begin{array}{r}\text { Before etanercept } \\
\qquad(n=10)\end{array}$ & $\begin{array}{r}\text { Before } \\
\text { placebo } \\
(n=10)\end{array}$ & $\begin{array}{r}\text { After etanercept } \\
\qquad(n=10)\end{array}$ & $\begin{array}{r}\text { After } \\
\text { placebo } \\
(n=10)\end{array}$ \\
\hline Sex (female/male) & $6 / 4$ & $7 / 3$ & - & - \\
\hline Age (years) & $72.6 \pm 2.6$ & $71.4 \pm 3.6$ & - & - \\
\hline BMI $\left(\mathrm{kg} / \mathrm{m}^{2}\right)$ & $23.6 \pm 3.4$ & $24.9 \pm 4.1$ & - & - \\
\hline $\begin{array}{l}\text { Blood pressure } \\
\text { (systolic/diastolic, } \mathrm{mmHg} \text { ) }\end{array}$ & $160 \pm 5 / 87 \pm 4$ & $159 \pm 4 / 87 \pm 5$ & - & - \\
\hline Smokers (n) & 2 & 3 & - & - \\
\hline Hypertension (n) & 6 & 3 & - & - \\
\hline Hypercholesterolemia ( $n$ ) & 3 & 2 & - & - \\
\hline $\begin{array}{l}\text { Physical activity level } \\
\text { before PMR onset } \\
\text { (1, high; } 2 \text {, medium; } 3 \text {, low) }\end{array}$ & $1.4 \pm 0.1$ & $1.9 \pm 0.2$ & - & - \\
\hline $\begin{array}{l}\text { Physical activity level } \\
\text { (1, high; } 2 \text {, medium; } 3 \text {, low) }\end{array}$ & $2.9 \pm 0.1$ & $2.9 \pm 0.1$ & $2.7 \pm 0.2$ & $3.0 \pm 0$ \\
\hline Morning stiffness (min) & $98 \pm 16$ & $117 \pm 19$ & $70 \pm 17$ & $103 \pm 21$ \\
\hline Patients' assessment of pain, VAS $(0-10 \mathrm{~cm})$ & $6.2 \pm 0.7$ & $5.0 \pm 0.8$ & $5.9 \pm 0.6$ & $5.4 \pm 0.5$ \\
\hline Cumulative intake of tramadol (tablets/14 days) & - & - & $47 \pm 8$ & $55 \pm 12$ \\
\hline
\end{tabular}

Data are mean \pm standard error of the mean. PMR, polymyalgia rheumatica; VAS, visual analog scale. 
Table 2 Characteristics of control subjects at baseline and after etanercept/placebo treatment

\begin{tabular}{|c|c|c|c|c|}
\hline & $\begin{array}{r}\text { Before etanercept } \\
(n=10)\end{array}$ & $\begin{array}{r}\text { Before } \\
\text { placebo } \\
(n=10)\end{array}$ & $\begin{array}{r}\text { After etanercept } \\
(n=10)\end{array}$ & $\begin{array}{r}\text { After } \\
\text { placebo } \\
(n=10) \\
\end{array}$ \\
\hline Sex (female/male) & $9 / 1$ & $6 / 4$ & - & - \\
\hline Age (years) & $69.9 \pm 1.2$ & $77.0 \pm 5.7$ & - & - \\
\hline BMI $\left(\mathrm{kg} / \mathrm{m}^{2}\right)$ & $25.5 \pm 0.9$ & $26.4 \pm 1.4$ & - & - \\
\hline $\begin{array}{l}\text { Blood pressure } \\
\text { (systolic/diastolic, } \mathrm{mmHg} \text { ) }\end{array}$ & $148 \pm 5 / 85 \pm 3$ & $150 \pm 4 / 85 \pm 2$ & - & - \\
\hline Smokers (n) & 2 & 3 & - & - \\
\hline Hypertension (n) & 5 & 4 & - & - \\
\hline Hypercholesterolemia (n) & 2 & 1 & - & - \\
\hline $\begin{array}{l}\text { Physical activity level } \\
\text { (1, high; } 2 \text {, medium; 3, low) }\end{array}$ & $* 1.5 \pm 0.2$ & $* 1.9 \pm 0.1$ & $* 1.6 \pm 0.2$ & $* 1.8 \pm 0.2$ \\
\hline $\operatorname{ESR}(\mathrm{mm} / \mathrm{hr})$ & ${ }^{*} 11.0 \pm 3.1$ & ${ }^{*} 9.1 \pm 1.3$ & *9.4 \pm 2.8 & ${ }^{*} 13.5 \pm 1.2$ \\
\hline CRP (mg/dl) & $* 1.1 \pm 0.1$ & ${ }^{*}<1.0$ & ${ }^{*}<1.0$ & ${ }^{*}<1.0$ \\
\hline Elevation of arms (3-0) & ${ }^{*} 0$ & ${ }^{*} 0$ & ${ }^{*} 0$ & ${ }^{*} 0$ \\
\hline Morning stiffness (min) & $* 6.0 \pm 2.0$ & ${ }^{*} 0$ & ${ }^{*} 0$ & ${ }^{*} 0$ \\
\hline Physician's global assessment, VAS $(0-10 \mathrm{~cm})$ & ${ }^{*} 0.2 \pm 0.2$ & ${ }^{*} 0$ & $*^{*} 0$ & ${ }^{*} 0$ \\
\hline Subjects' assessment of pain, VAS $(0-10 \mathrm{~cm})$ & $*^{*} 0.75 \pm 0.5$ & $* 1.0 \pm 0.4$ & ${ }^{*} 0.06 \pm 0.1$ & ${ }^{*} 0$ \\
\hline
\end{tabular}

Data are mean \pm standard error of the mean.

CRP, C-reactive protein; ESR, erythrocyte sedimendation rate; VAS, visual analog scale.

*Values significantly different from values in patients with polymyalgia rheumatica $(P<0.001$ to 0.01$)$.

statistically significant, and functional status as evaluated by HAQ did not improve in any of the groups.

In order to verify that the anti-TNF- $\alpha$ treatment was still effective at the final evaluation by day 15 , that is, three days after the last etanercept injection, and that potential effects of the blockade accordingly were not underestimated, plasma TNF- $\alpha$ concentrations were determined. However, instead of being reduced, in etanercept-treated

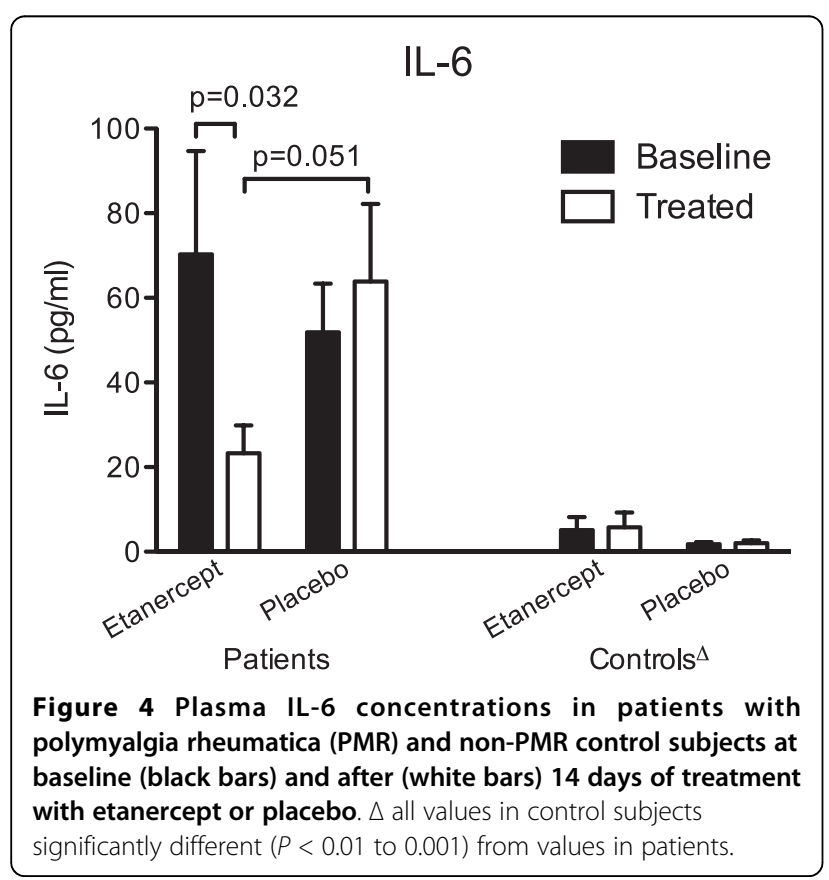

patients the TNF- $\alpha$ concentrations were higher than before treatment and also higher than in placebo-treated patients (Figure 5). The increased TNF- $\alpha$ values observed after etanercept treatment were probably due to the simultaneous detection of both free and accumulated etanercept-bound TNF- $\alpha$ [27]. In agreement with this view, during etanercept treatment of control subjects TNF- $\alpha$ concentrations were also markedly increased (Figure 5).

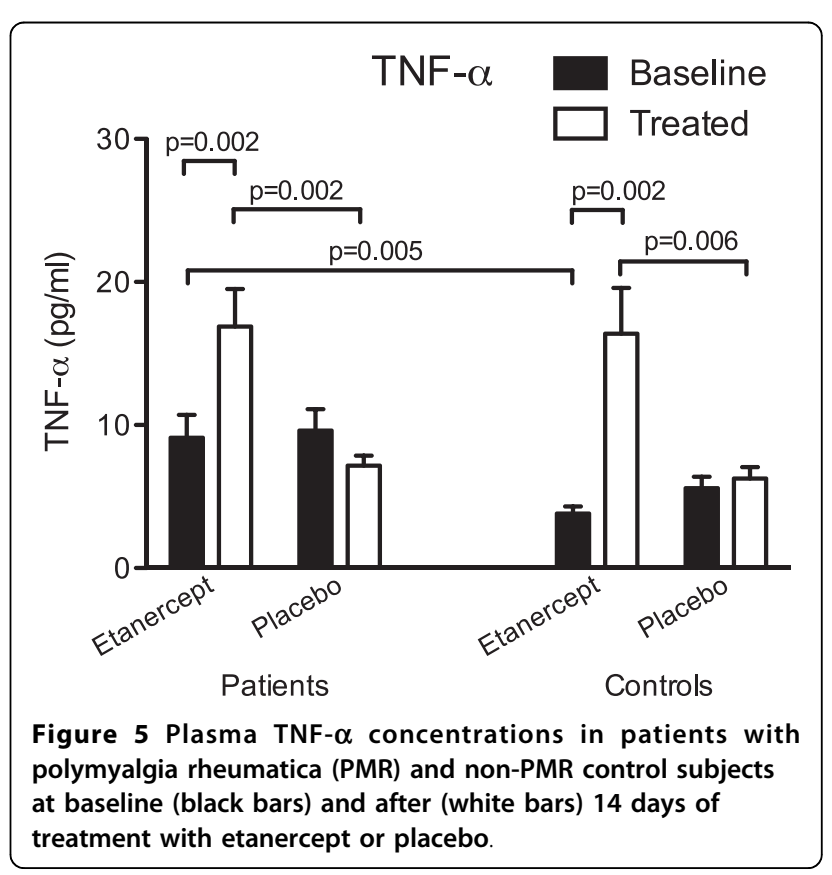


Furthermore, in patients treated with etanercept, TNF$\alpha$ concentrations were reduced by $61 \%$ after IgG depletion of plasma. This process also removes etanercept (free etanercept as well as etanercept-TNF- $\alpha$ complexes), which contains the Fc fragment of IgG1. TNF- $\alpha$ concentrations were similar in IgG-depleted plasma from etanercept-treated compared with placebo-treated patients. As some etanercept-bound TNF- $\alpha$ will remain in IgG-depleted plasma from etanercept-treated patients, while only free TNF- $\alpha$ is present in IgG-depleted plasma from placebo-treated patients, the similar overall TNF- $\alpha$ concentrations in IgG-depleted plasma most likely reflect that free TNF- $\alpha$ concentrations were lower in plasma from etanercept-treated patients than in plasma from placebo-treated patients. So, these observations indicate that by the end of the trial, etanercept was still present in plasma and able to lower free TNF- $\alpha$ concentrations compared with values in placebo-treated patients [27].

In the present study, TNF- $\alpha$ concentrations in plasma were higher in patients with PMR than in non-PMR controls subjects (Figure 2) before treatment in both treatment groups. This is in accordance with findings in a recent study, in which we showed that interstitial TNF- $\alpha$ concentrations in affected muscle are increased in untreated PMR and normalized in parallel with complete clinical remission during 14 days of prednisolone therapy [9]. Although these findings are suggestive, the fact that in the present study TNF- $\alpha$ blockade had only a modest limiting effect on disease activity indicates that TNF- $\alpha$ is not a major determinant of the pathophysiology in PMR. Interestingly, in a study of two patients with previously untreated GCA, a condition generally considered to be closely related to PMR, a dramatic improvement was observed within 14 days of monotherapy with the anti-TNF- $\alpha$ monoclonal antibody infliximab, and it was concluded that in this disease TNF- $\alpha$ plays a major role in mediating inflammation [28].

In the present study, the same experienced rheumatology specialist evaluated all patients. It is interesting to note that although being blinded to treatment allocation, the specialist's evaluation of disease activity as reflected by shoulder mobility (E) and $\mathrm{VAS}_{\mathrm{ph}}$, was in better agreement with paraclinical measures (ESR, CRP, and IL-6) than the patients' own evaluations of disease activity as apparent from MST, $\mathrm{VAS}_{\mathrm{p}}$, and HAQ. Due to the monotherapeutic design of the present RCT, potential effects of etanercept were not obscured by simultaneous administration of other anti-inflammatory drugs such as GCs. As a consequence, however, the trial had to be brief. Thus, it is possible that a larger effect of etanercept would have been demonstrated had it been possible to extend the duration of the trial. However, in patients with RA the effect of etanercept occurs rapidly [20]. Furthermore, in patients with GCA a small uncontrolled study showed a dramatic effect of infliximab monotherapy treatment in the early phase [28]. Conversely, a RCT has shown that extension of infliximab therapy to 22 weeks does not diminish the need for simultaneous GC therapy [29]. Still, in some contrast to the latter study, a GC-sparing effect of etanercept has been demonstrated in a recent $\mathrm{RCT}$ when administered late in the course of GCA in patients with side effects from GC [19]. However, such patients may have a particularly strong inflammatory reaction making them relatively GC-resistant $[6,30]$ and sensitive to TNF- $\alpha$ blockade [31].

The disease ameliorating effect of etanercept seen in the present study contrasts with the lack of benefit of infliximab found in a RCT with GC-treated, newly diagnosed patients with PMR [15]. This discrepancy may reflect differences in study design or differences in mechanism of action of the two TNF- $\alpha$ antagonists. Arguing in favor of the latter possibility, in a small, uncontrolled study, it was demonstrated that etanercept was beneficial in the treatment of GC-treated patients with newly diagnosed PMR and decompensated diabetes [18]. However, arguing against the latter possibility, in line with our findings, infliximab monotherapy induced remission in a small, uncontrolled study with newly diagnosed patients with PMR [17]. Moreover, in another uncontrolled study of a small number of patients with longstanding, GC-resistant PMR, administration of infliximab was accompanied by diminished disease activity [32].

Overall, current evidence indicates that the effect of TNF- $\alpha$ blockade in PMR and GCA is moderate. For that reason and considering the high cost, TNF- $\alpha$ therapy in PMR and GCA seems justified solely when these diseases can only be controlled by GC doses that are untenable due to serious adverse effects and comorbidities induced or worsened by steroids.

\section{Conclusions}

In conclusion, the present $\mathrm{RCT}$ has demonstrated that etanercept monotherapy ameliorates disease activity in newly diagnosed, GC-naïve patients with PMR. However, the effect is modest, indicating that TNF- $\alpha$ does not play a central role in the pathophysiology of PMR.

\section{Abbreviations}

BMI: body mass index; CRP: C-reactive protein; CV: coefficient of variation; ESR: erythrocyte sedimentation rate; GC: glucocorticoid; GCA: giant cell arteritis; HAQ: health assessment questionaire; Ig: immunoglobulin; IL: interleukin; MST: morning stiffness; PMR: polymyalgia rheumatica; PMR-AS: PMR activity score; RA: rheumatoid arthritis; RCT: randomized controlled trial; TNF: tumor necrosis factor; VAS: visual analog score; VAS: patient's visual analog score; $V_{\text {AS }}$ : physician's visual analog score. 


\section{Acknowledgements}

Lisbeth Kall is thanked for skilled technical assistance. The GCP-unit at the University of Copenhagen is thanked for their collaboration. Senior nurses Kirsten Gleerup and Marianne Tørper are thanked for their assistance with trial medication administration and preparation. The study was supported by grants from The Danish Rheumatism Association (grant number 233-46314.10.05) and by The Danish Medical Research Council (grant number 27106-0311).

\section{Authors' contributions}

FK and HG contributed equally to the planning and conduct of the trial as well as to the analysis and interpretation of study outcomes. Both authors drafted and approved the manuscript.

\section{Competing interests}

The authors declare that they have no competing interests.

Received: 8 April 2010 Revised: 15 July 2010

Accepted: 20 September 2010 Published: 20 September 2010

\section{References}

1. Salvarani C, Cantini F, Hunder GG: Polymyalgia rheumatica and giant-cell arteritis. Lancet 2008, 372:234-245.

2. Salvarani C, Barozzi L, Cantini F, Niccoli L, Boiardi L, Valentino M, Pipitone N Bajocchi G, Macchioni P, Catanoso MG, Olivieri I, Hunder GG: Cervical interspinous bursitis in active polymyalgia rheumatica. Ann Rheum Dis 2008, 67:758-761.

3. Healey LA: Polymyalgia rheumatica is the result of synovitis. J Clin Rheumatol 2006, 12:165-166.

4. Cantini F, Niccoli L, Nannini C, Padula A, Olivieri I, Boiardi L, Salvarani C: Inflammatory changes of hip synovial structures in polymyalgia rheumatica. Clin Exp Rheumatol 2005, 23:462-468.

5. Martinez-Taboada VM, Alvarez L, RuizSoto M, Marin-Vidalled MJ, LopezHoyos M: Giant cell arteritis and polymyalgia rheumatica: role of cytokines in the pathogenesis and implications for treatment. Cytokine 2008, 44:207-220.

6. Hernandez-Rodriguez J, Garcia-Martinez A, Casademont J, Filella X, Esteban MJ, Lopez-Soto A, Fernandez-Sola J, Urbano-Marquez A, Grau JM, Cid MC: A strong initial systemic inflammatory response is associated with higher corticosteroid requirements and longer duration of therapy in patients with giant-cell arteritis. Arthritis Rheum 2002, 47:29-35.

7. Uceyler N, Schafers M, Sommer C: Mode of action of cytokines on nociceptive neurons. Exp Brain Res 2009, 196:67-78.

8. Uceyler N, Sommer C: Cytokine regulation in animal models of neuropathic pain and in human diseases. Neurosci Lett 2008, 437:194-198.

9. Kreiner F, Galbo H: Increased muscle interstitial levels of inflammatory cytokines in polymyalgia rheumatica. Arthritis Rheum 2010.

10. Salvarani C, Cantini F, Boiardi L, Hunder GG: Polymyalgia rheumatica and giant-cell arteritis. N Engl J Med 2002, 347:261-271.

11. Gabriel SE, Sunku J, Salvarani C, O'Fallon WM, Hunder GG: Adverse outcomes of antiinflammatory therapy among patients with polymyalgia rheumatica. Arthritis Rheum 1997, 40:1873-1878.

12. Cutolo M, Montecucco CM, Cavagna L, Caporali R, Capellino S, Montagna P, Fazzuoli L, Villaggio B, Seriolo B, Sulli A: Serum cytokines and steroidal hormones in polymyalgia rheumatica and elderly-onset rheumatoid arthritis. Ann Rheum Dis 2006, 65:1438-1443.

13. Nothnagl T, Leeb BF: Diagnosis, differential diagnosis and treatment of polymyalgia rheumatica. Drugs Aging 2006, 23:391-402.

14. van Vollenhoven RF: Treatment of rheumatoid arthritis: state of the art 2009. Nat Rev Rheumatol 2009, 5:531-541.

15. Salvarani $C$, Macchioni $P$, Manzini C, Paolazzi G, Trotta A, Manganelli $P$ Cimmino M, Gerli R, Catanoso MG, Boiardi L, Cantini F, Klersy C, Hunder GG Infliximab plus prednisone or placebo plus prednisone for the initial treatment of polymyalgia rheumatica: a randomized trial. Ann Intern Med 2007, 146:631-639

16. Keystone EC: Switching tumor necrosis factor inhibitors: an opinion. Nat Clin Pract Rheumatol 2006, 2:576-577.

17. Migliore A, Massafra U, Carloni E, Padalino C, Martin Martin S, Lasaracina F, Dibiase N, Alimonti A, Granata M: TNF-alpha blockade induce clinical remission in patients affected by polymyalgia rheumatica associated to diabetes mellitus and/or osteoporosis: a seven cases report. Eur Rev Med Pharmacol Sci 2005, 9:373-378.

18. Corrao S, Pistone G, Scaglione R, Colomba D, Calvo L, Licata G: Fast recovery with etanercept in patients affected by polymyalgia rheumatica and decompensated diabetes: a case-series study. Clin Rheumatol 2009, 28:89-92.

19. Martinez-Taboada VM, Rodriguez-Valverde V, Carreno L, Lopez-Longo J, Figueroa M, Belzunegui J, Mola EM, Bonilla G: A double-blind placebo controlled trial of etanercept in patients with giant cell arteritis and corticosteroid side effects. Ann Rheum Dis 2008, 67:625-630.

20. Weinblatt ME, Kremer JM, Bankhurst AD, Bulpitt KJ, Fleischmann RM, Fox Rl, Jackson CG, Lange $M$, Burge DJ: A trial of etanercept, a recombinant tumor necrosis factor receptor:Fc fusion protein, in patients with rheumatoid arthritis receiving methotrexate. N Engl J Med 1999 340:253-259.

21. Chuang TY, Hunder GG, Ilstrup DM, Kurland LT: Polymyalgia rheumatica: a 10-year epidemiologic and clinical study. Ann Intern Med 1982, 97:672-680.

22. Randomization.com. [http://randomization.com]

23. Thorsen H, Hansen TM, McKenna SP, Sorensen SF, Whalley D: Adaptation into Danish of the Stanford Health Assessment Questionnaire (HAQ) and the Rheumatoid Arthritis Quality of Life Scale (RAQoL). Scand J Rheumatol 2001, 30:103-109.

24. Leeb BF, Bird HA: A disease activity score for polymyalgia rheumatica. Ann Rheum Dis 2004, 63:1279-1283.

25. Binard A, de Bandt M, Berthelot JM, Saraux A: Performance of the polymyalgia rheumatica activity score for diagnosing disease flares. Arthritis Rheum 2008, 59:263-269.

26. Binard A, Lefebvre B, De Bandt M, Berthelot JM, Saraux A: Validity of the polymyalgia rheumatica activity score in primary care practice. Ann Rheum Dis 2009, 68:541-545.

27. Nowlan ML, Drewe E, Bulsara H, Esposito N, Robins RA, Tighe PJ, Powell RJ, Todd I: Systemic cytokine levels and the effects of etanercept in TNF receptor-associated periodic syndrome (TRAPS) involving a C33Y mutation in TNFRSF1A. Rheumatology (Oxford) 2006, 45:31-37.

28. Andonopoulos AP, Meimaris N, Daoussis D, Bounas A, Giannopoulos G. Experience with infliximab (anti-TNF alpha monoclonal antibody) as monotherapy for giant cell arteritis. Ann Rheum Dis 2003, 62:1116.

29. Hoffman GS, Cid MC, Rendt-Zagar KE, Merkel PA, Weyand CM, Stone JH, Salvarani C, Xu W, Visvanathan S, Rahman MU: Infliximab for maintenance of glucocorticosteroid-induced remission of giant cell arteritis: a randomized trial. Ann Intern Med 2007, 146:621-630.

30. Hernandez-Rodriguez J, Segarra M, Vilardell C, Sanchez M, GarciaMartinez A, Esteban MJ, Queralt C, Grau JM, Urbano-Marquez A, Palacin A, Colomer D, Cid MC: Tissue production of pro-inflammatory cytokines (IL-1beta, TNFalpha and IL-6) correlates with the intensity of the systemic inflammatory response and with corticosteroid requirements in giant-cell arteritis. Rheumatology (Oxford) 2004, 43:294-301.

31. Salvarani C, Pipitone N, Boiardi L, Hunder GG: Do we need treatment with tumour necrosis factor blockers for giant cell arteritis? Ann Rheum Dis 2008, 67:577-579.

32. Salvarani C Cantini F, Niccoli L, Catanoso MG, Macchioni P, Pulsatelli L, Padula A, Olivieri I, Boiardi L: Treatment of refractory polymyalgia rheumatica with infliximab: a pilot study. J Rheumatol 2003, 30:760-763.

\section{doi:10.1186/ar3140}

Cite this article as: Kreiner and Galbo: Effect of etanercept in polymyalgia rheumatica: a randomized controlled trial. Arthritis Research \& Therapy 2010 12:R176. 